ARTICLE

\title{
Perspective on the phase diagram of cuprate high-temperature superconductors
}

Damian Rybicki ${ }^{1,2}$, Michael Jurkutat ${ }^{1}$, Steven Reichardt ${ }^{1}$, Czesław Kapusta $^{2}$ \& Jürgen Haase ${ }^{1}$

Universal scaling laws can guide the understanding of new phenomena, and for cuprate high-temperature superconductivity the influential Uemura relation showed, early on, that the maximum critical temperature of superconductivity correlates with the density of the superfluid measured at low temperatures. Here we show that the charge content of the bonding orbitals of copper and oxygen in the ubiquitous $\mathrm{CuO}_{2}$ plane, measured with nuclear magnetic resonance, reproduces this scaling. The charge transfer of the nominal copper hole to planar oxygen sets the maximum critical temperature. A three-dimensional phase diagram in terms of the charge content at copper as well as oxygen is introduced, which has the different cuprate families sorted with respect to their maximum critical temperature. We suggest that the critical temperature could be raised substantially if one were able to synthesize materials that lead to an increased planar oxygen hole content at the expense of that of planar copper.

\footnotetext{
${ }^{1}$ Institute of Experimental Physics II, University of Leipzig, Faculty of Physics and Earth Sciences, Linnéstrasse 5, Leipzig 04103, Germany. ${ }^{2}$ AGH University of Science and Technology, Faculty of Physics and Applied Computer Science, Department of Solid State Physics, al. A. Mickiewicza 30, Krakow 30-059, Poland. Correspondence and requests for materials should be addressed to D.R. (email: ryba@agh.edu.pl).
} 
$\mathrm{T}$ he understanding of the complex properties of the cuprates, and what causes their high critical temperature of superconductivity $\left(T_{\mathrm{c}}\right)$, is one of the greatest challenges in condensed matter physics. From it one expects clues that make the synthesis of cuprates with much higher $T_{\mathrm{c}}$ possible or of how to improve other fundamental properties. In particular, it is still not well understood what sets the very different, maximum $T_{\mathrm{c}}$ 's for different families of materials. An early experimental observation in this regard is the famous Uemura plot $^{1}$. It shows that the maximum $T_{\mathrm{c}}$ is correlated with the muon spin relaxation rate $\sigma_{0}$ (extrapolated to $T=0 \mathrm{~K}$ ) that is proportional to the superfluid density divided by the effective mass $\left(\sigma_{0} \propto n_{\mathrm{s}} / \mathrm{m}^{*}\right)$. This relation holds for the underdoped materials and orders different cuprate families with respect to their maximum $T_{\mathrm{c}}$. The Uemura relation and subsequent scaling laws have remained stimulating, up to now, and some were shown to be valid for other superconductors as well ${ }^{2-9}$. While there are various attempts at a theoretical explanation of the Uemura relation ${ }^{4,10-12}$, a connection to other experimentally probed properties of cuprates is still lacking, in particular to material chemistry parameters.

The various cuprate families have in common, see Fig. 1a, a $\mathrm{CuO}_{2}$ plane and charge reservoir (CR) layers that separate the planes from each other. While the nearly square $\mathrm{CuO}_{2}$ plane, defined by the $\mathrm{Cu} 3 d_{x^{2}-y^{2}}$ orbital bonding to four $\mathrm{O} 2 p_{\sigma}$ orbitals, is very similar for all systems, the $\mathrm{CR}$ chemistry can vary significantly. The nominal hole at $\mathrm{Cu}\left(3 d^{9}\right.$ configuration) is responsible for strong magnetic correlations that make parent materials antiferromagnetic. Holes or electrons can be added to the $\mathrm{CuO}_{2}$ plane by alteration of $\mathrm{CR}$ layers. As a result, static magnetism vanishes and new electronic phenomena emerge and the systems become conducting or superconducting. There are many similarities between different cuprate families, and one typically differentiates only between the hole and electron-doped phase diagrams, depicted in Fig. 1b, that appear to show a distinct asymmetry. However, the extent in temperature and doping of the different phases and observed phenomena varies substantially between different families, also for the much more thoroughly investigated hole doped materials. In addition, the comparability between different families is somewhat obstructed with regard to doping. For example, while for $\mathrm{La}_{2}{ }_{-} \mathrm{Sr}_{x} \mathrm{CuO}_{4}$ the doping level can be varied over a large range quite reliably by stoichiometry, interstitial doping with oxygen $\left(\mathrm{O}_{\delta}\right)$ as, for example, in $\mathrm{HgBa}_{2} \mathrm{CuO}_{4+\delta}$ leaves uncertainties with regard to the actual doping level. In addition, ionic migration may cause phase separation or other ordering phenomena as in the $\mathrm{YBa}_{2} \mathrm{Cu}_{3} \mathrm{O}_{6+y}$ systems. Given the very different $T_{\mathrm{c}}$ for various cuprate families, it was questioned whether the average doping level of the $\mathrm{CuO}_{2}$ plane is the appropriate chemical parameter for discussing all aspects of the complex physical properties of the cuprates, or whether other parameters should be considered as well, for example, distances within the plane, buckling, disorder, the role of the apical oxygen, or interlayer coupling. Nevertheless, from the understanding of the electronic properties we expect, in particular, clues as how to raise $T_{\mathrm{c}}$.

Nuclear magnetic resonance (NMR), as a local probe of the magnetic spin susceptibility, focusses mostly on measurements of shift and relaxation caused by the interaction of the nuclear magnetic dipole moment with electronic magnetic moments. However, the nuclear electric quadrupole moment for nuclei with spin $I>1 / 2\left(I=3 / 2\right.$ for ${ }^{63,65} \mathrm{Cu}, I=5 / 2$ for $\left.{ }^{17} \mathrm{O}\right)$ interacts with the local electric field gradient (EFG) causing a quadrupole splitting $\left(v_{\mathrm{Q}}\right)$ of the NMR lines in high magnetic fields. The EFG at the nuclear site is very sensitive to the local charge symmetry, and has been useful for assigning NMR signals to the various lattice positions or detecting inhomogeneous charge distributions in the $\mathrm{CuO}_{2}$ plane ${ }^{13,14}$. Since the quadrupole splittings of planar $\mathrm{Cu}$ and $\mathrm{O}$ depend on doping, models have been put forward that attempted to understand these changes in terms of the hole content of certain orbitals, see for example, refs 15-18 and works a

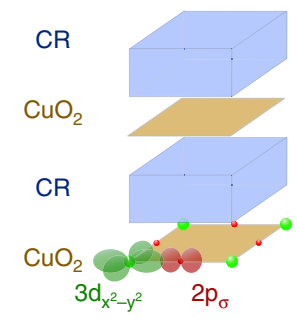

Parent: $n_{d}+2 n_{p}=1$

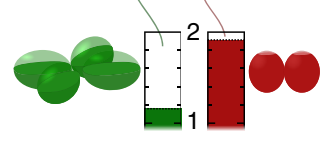

b

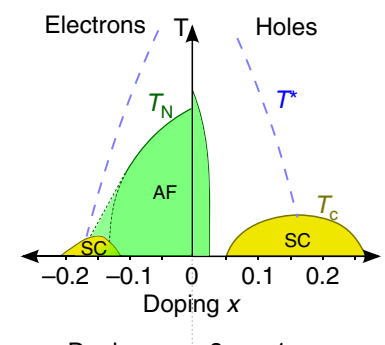

Doping: $n_{d}+2 n_{p}=1+x$

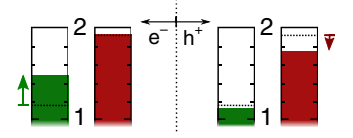

C

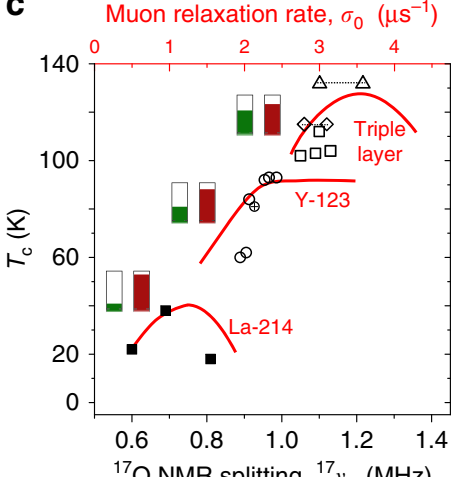

${ }^{17} \mathrm{O}$ NMR splitting, ${ }^{17} v_{\mathrm{Q}}(\mathrm{MHz})$ $\mu \mathrm{SR}:$
- Uemuta - Uemura
NMR:

$\triangle \mathrm{Pr}-214 \quad \star \mathrm{Hg}-120$ $\checkmark \mathrm{Nd}-214$ म $\mathrm{Tl}-2201$ d

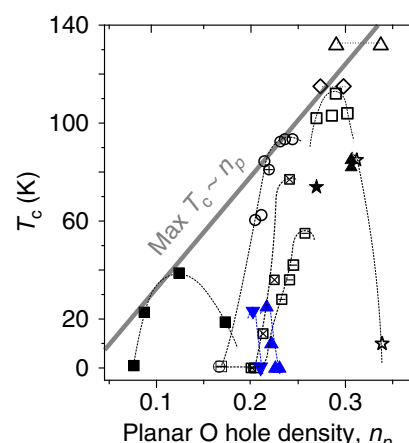

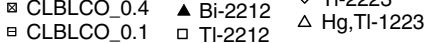

Figure 1 | General features of the cuprates. (a) The cuprates' layered structure consists of $\mathrm{CR}$ layers and $\mathrm{CuO}_{2}$ planes with the bonding orbitals $\mathrm{Cu} 3 d_{x^{2}-y^{2}}$ and $\mathrm{O} 2 p_{\sigma}$, which share the nominal $3 d$ hole of the $\mathrm{Cu}^{2+}$ ion. Columns indicate occupation of Cu $3 d_{x^{2}}-y^{2}\left(2-n_{d}\right)$ and O $2 p_{\sigma}\left(2-n_{p}\right)$, with hole contents $n_{d}$ and $n_{p}$ measurable with NMR. (b) Schematic representation of electronic phase diagram of the cuprates for electron and hole doping $x$ : AF phase below Néel temperature $\left(T_{N}\right)$, SC below critical temperature $\left(T_{c}\right)$, and pseudogap regime below pseudogap temperature $\left(T^{\star}\right)$. Doped electrons ( $\left.e^{-}\right)$ go to the $\mathrm{Cu} 3 d_{x^{2}-y^{2}}$ orbital almost exclusively, while doped holes $\left(\mathrm{h}^{+}\right)$predominantly go to the $\mathrm{O} 2 p_{\sigma}$ orbital, arrows next to columns indicate changes of $n_{d}$ and $n_{p}$ caused by doping. (c) Solid red: Uemura plot ${ }^{1}$, that is, $T_{c}$ versus muon spin relaxation rate $\left(\sigma_{0}\right.$, upper abscissa); black symbols: $T_{c}$ versus planar oxygen quadrupole splitting ${ }^{17} v_{\mathrm{Q}}$ (lower abscissa). For list of abbreviations see Table 1. For triple layer Tl-2223 and $\mathrm{Hg}$, Tl- 1223 the pairs connected with a dotted line belong to the same sample and correspond to planar $\mathrm{O}$ sites of inner and outer layer (smaller splitting corresponds to underdoped inner $\mathrm{CuO}_{2}$ layer). (d) $T_{c}$ versus planar $\mathrm{O}$ hole density $n_{p}$ calculated from ${ }^{17} v_{\mathrm{Q}}$ for all available data (see text). Black dotted lines are guides to the eye and connect different doping levels for one family. Solid grey line indicates increase of the maximum $T_{c}$ (that is, for the optimal doping level) as a function of $n_{p}$. $\mathrm{AF}$, antiferromagnetic; $\mathrm{SC}$, superconducting phase. 
cited therein. While trends relating the local charge distribution with $T_{\mathrm{c}}$ could be established, the uncertainty with regard to the EFG contributions from a variable $\mathrm{CR}$ chemistry, limited quantitative agreement with charge contents expected from stoichiometry, as well as insufficient experimental data hampered advances of such analyses.

Here we show that if we plot $T_{\mathrm{c}}$ versus the planar ${ }^{17} \mathrm{O}$ NMR quadrupole splitting, a functional dependence very similar to that of the Uemura plot emerges. This documents that the superfluid density is a function of the EFG at planar O. Based on very recent progress in the understanding of NMR quadrupole splittings in terms of the charge distribution in the $\mathrm{CuO}_{2}$ plane ${ }^{19}$ we show that the maximum $T_{\mathrm{c}}$ increases with the hole content of the planar $\mathrm{O} 2 p_{\sigma}$ orbital, at the expense of that at $\mathrm{Cu} 3 d_{x^{2}-y^{2}}$. Thus, we identify material chemistry parameters, the hole contents at planar $\mathrm{Cu}$ and $\mathrm{O}$, that are largely temperature independent, yet determine the superfluid density at low temperatures. This finding stimulates the use of these orbital hole contents, calculated from NMR literature data ${ }^{16,18-49}$, to draw a threedimensional cuprate phase diagram that encompasses all cuprate families and has the superconducting domes ordered according to the maximum $T_{\mathrm{c}}$. We argue that such a phase diagram might be very useful in discussing the complex properties of the cuprates.

\section{Results}

Superfluid density and charge densities in the $\mathrm{CuO}_{2}$ plane. We plot in Fig. 1c, together with the original Uemura plot (in red), $T_{\mathrm{c}}$ versus ${ }^{17} v_{\mathrm{Q}}$ for similar materials and doping, and find a striking correspondence. This shows that the muon spin relaxation rate deep inside the superconducting state must be tied to the almost temperature independent EFG at the planar $\mathrm{O}$ nucleus, which determines the ${ }^{17} \mathrm{O}$ NMR splitting measured far above $T_{\mathrm{c}}$, a rather unanticipated result.

It was confirmed recently, based on NMR data on the electron-doped and parent compounds, that NMR quadrupole splittings provide a quantitative measure of the charge distribution in the $\mathrm{CuO}_{2}$ plane of apparently all cuprates ${ }^{19}$. A list of materials with abbreviations is given in Table 1. It was shown that the hole densities in the $\mathrm{Cu} 3 d_{x^{2}-y^{2}}$ orbital $\left(n_{d}\right)$ and the $\mathrm{O} 2 p_{\sigma}$ orbital $\left(n_{p}\right)$ in the $\mathrm{CuO}_{2}$ plane are related to the experimentally measured splittings ${ }^{63} v_{\mathrm{Q}}$ at ${ }^{63} \mathrm{Cu}$ and ${ }^{17} v_{\mathrm{Q}}$ at ${ }^{17} \mathrm{O}$ as follows ${ }^{18,19}$ :

$$
\begin{gathered}
{ }^{17} v_{\mathrm{Q}}=2.45 \mathrm{MHz} \cdot n_{p}+0.39 \mathrm{MHz}, \\
{ }^{63} v_{\mathrm{Q}}=94.3 \mathrm{MHz} \cdot n_{d}-5.68 \mathrm{MHz} \cdot\left(8-4 n_{p}\right) .
\end{gathered}
$$

The planar oxygen splitting in equation (1) is only dependent on the hole content $n_{p}$ of the onsite bonding orbital $2 p_{\sigma}$ with the prefactor $2.45 \mathrm{MHz}$ derived from the electric hyperfine interaction experimentally determined with atomic spectroscopy

\begin{tabular}{|c|c|}
\hline Abbreviation & Formula \\
\hline $\mathrm{Bi}-2212$ & $\mathrm{Bi}_{2} \mathrm{Sr}_{2} \mathrm{CaCu}_{2} \mathrm{O}_{8+\delta}$ \\
\hline CLBLCO_x & $\left(\mathrm{Ca}_{x} \mathrm{La}_{1-x}\right)\left(\mathrm{Ba}_{1.75-x} \mathrm{La}_{0.25+x}\right) \mathrm{Cu}_{3} \mathrm{O}_{6+y}$ \\
\hline $\mathrm{Hg}-1201$ & $\mathrm{HgBa}_{2} \mathrm{CuO}_{4+\delta}$ \\
\hline $\mathrm{Hg}$, TI-1223 & $\mathrm{Hg}_{0.5} \mathrm{TI}_{0.5} \mathrm{Ba}_{2}\left(\mathrm{Ca}_{1-x} \mathrm{Sr}_{x}\right)_{2} \mathrm{Cu}_{3} \mathrm{O}_{8+\delta}$ \\
\hline La-214 & $\mathrm{La}_{2-x} \mathrm{Sr}_{x} \mathrm{CuO}_{4}$ \\
\hline $\mathrm{Nd}-214$ & $\mathrm{Nd}_{2-x} \mathrm{Ce}_{x} \mathrm{CuO}_{4}$ \\
\hline $\operatorname{Pr}-214$ & $\mathrm{Pr}_{2-x} \mathrm{Ce}_{x} \mathrm{CuO}_{4}$ \\
\hline TI-2201 & $\mathrm{Tl}_{2} \mathrm{Ba}_{2} \mathrm{CuO}_{y}$ \\
\hline TI-2212 & $\mathrm{Tl}_{2} \mathrm{Ba}_{2} \mathrm{CaCu}_{2} \mathrm{O}_{8-\delta}$ \\
\hline TI-2223 & $\mathrm{Tl}_{2} \mathrm{Ba}_{2} \mathrm{Ca}_{2} \mathrm{Cu}_{3} \mathrm{O}_{10}-\delta$ \\
\hline Y-123 & $\mathrm{YBa}_{2} \mathrm{Cu}_{3} \mathrm{O}_{6+y}$ \\
\hline Y-124 & $\mathrm{YBa}_{2} \mathrm{Cu}_{4} \mathrm{O}_{8}$ \\
\hline
\end{tabular}

Table 1 | List of abbreviations. of the $\mathrm{O} 2 p^{5}$ state $^{18}$. The term of $0.39 \mathrm{MHz}$ is due to the charge symmetry at planar oxygen in the ubiquitous $\mathrm{CuO}_{2}$ plane, and this term is found to be rather independent on doping and similar for all families ${ }^{19}$. Therefore, one can easily convert the experimentally measured ${ }^{17} v_{\mathrm{Q}}$ into a reliable hole content $n_{p}$. The situation is somewhat more complicated for the $\mathrm{Cu}$ splitting in equation (2), which depends on the hole densities of both bonding orbitals. The first term is from the onsite hole content $n_{d}$ of $3 d_{x^{2}-y^{2}}$ with the prefactor $94.3 \mathrm{MHz}$, again derived from atomic spectroscopy of $\mathrm{Cu} 3 d^{9}$ state $^{18}$. The second term in equation (2) accounts for the EFG at the $\mathrm{Cu}$ nucleus caused by the charge in the bonding orbitals of the four surrounding planar $\mathrm{O}$ atoms, with the prefactor derived from the orbital overlap of $\mathrm{O} 2 p_{\sigma}$ with the empty $\mathrm{Cu} 4 p$ and the electric hyperfine interaction of the latter ${ }^{18}$.

First, we use equation (1) and convert all the planar oxygen splittings from the literature to $n_{p}$. The result is plotted in Fig. $1 \mathrm{~d}$, that is, we plot $T_{\mathrm{c}}$ versus $n_{p}$ for the different materials. Of course, this plot is very similar to Fig. 1c, but it includes non-superconducting underdoped and parent materials with $T_{\mathrm{c}}=0 \mathrm{~K}$. We see that different cuprate families have rather different $n_{p}$, which results in the sorting of the families as in the Uemura plot. We also recognize that a large $n_{p}$ is a prerequisite for a high maximum $T_{\mathrm{c}}$, that is, at optimal doping. In Fig. 1d one can also notice a parabolic-like dependence of $T_{\mathrm{c}}$ on the oxygen charge $n_{p}$, which resembles the typical phase diagram that shows a dome-like dependence of $T_{\mathrm{c}}$ on the average doping level. The correlation between $\sigma_{0}$ and ${ }^{17} v_{\mathrm{Q}}$ is lost in the overdoped regime where $\sigma_{0}$ decreases with increasing doping ${ }^{50,51}$, which was attributed to a decrease of $n_{s}$ (ref. 52).

In Fig. 1d, we also included recent results for the electron-doped materials ${ }^{19}$. For $\mathrm{Nd}_{1.85} \mathrm{Ce}_{0.15} \mathrm{CuO}_{4}$ the superfluid density was reported to be very similar to that of hole doped $\mathrm{YBa}_{2} \mathrm{Cu}_{3} \mathrm{O}_{6+y}$, albeit measured optically and not by muon spin relaxation $3,53,54$. We find that these results are also in agreement with ${ }^{17} v_{\mathrm{Q}}$ splittings (see Supplementary Fig. 1) and corresponding hole contents for those two families, cf. Fig. 1d. Electron doping appears to be less efficient in providing a high $T_{\mathrm{c}}$, but the rather high oxygen hole contents of the parent materials $\mathrm{Pr}_{2} \mathrm{CuO}_{4}$ and $\mathrm{Nd}_{2} \mathrm{CuO}_{4}$ suggest that hole doping should result in much higher $T_{c}$. The so-called infinite layer cuprate $\mathrm{Sr}_{1-x} \mathrm{La}_{x} \mathrm{CuO}_{2}$, for which there are no reports of ${ }^{17} \mathrm{O}$ splittings, has the highest $T_{\mathrm{c}}$ among electron-doped families and a very high muon spin relaxation rate $\left(\sigma_{0} \approx 4.5 \mu \mathrm{s}^{-1}\right)$ (ref. 53), and we expect a high $n_{p}$. Indeed, a rather high $T_{c}$ of more than $100 \mathrm{~K}$ was reported in the infinite layer system upon hole doping 55,56 .

Clearly, a large $n_{p}$ is a prerequisite for a high $T_{\mathfrak{c}}$, but is not sufficient, as expected for such a material chemistry parameter. If this empirical relation $\left(\max T_{\mathrm{c}} \propto n_{p}\right)$ remains valid for higher oxygen hole content, the $T_{\mathrm{c}}$ of the cuprates might be raised substantially by the proper chemistry (we estimate $300-400 \mathrm{~K}$ per oxygen hole from the straight line in Fig. 1d).

The splittings of the ${ }^{63} \mathrm{Cu}$ NMR lines can only be converted into $n_{d}$ if there are also ${ }^{17} \mathrm{O}$ NMR data available, cf. equation (2). However, there are much less ${ }^{17} \mathrm{O}$ splittings reported since the materials have to be enriched with ${ }^{17} \mathrm{O}$ (the naturally abundant ${ }^{16} \mathrm{O}$ nucleus has spin $I=0$ ) and therefore only part of ${ }^{63} \mathrm{Cu}$ splittings can be converted. (In Supplementary Fig. 1, we plot $T_{\mathrm{c}}$ versus experimentally measured splittings).

Note that the simple analysis using equations (1) and (2) gives hole densities that are in astonishingly good quantitative agreement with the total charge in the $\mathrm{CuO}_{2}$ plane expected from the stoichiometry of the materials ${ }^{19}$, that is,

$$
1+x=n_{d}+2 n_{p},
$$

where the factor of 2 accounts for the two $\mathrm{O}$ atoms per $\mathrm{CuO}_{2}$. 
This means that the sum of the hole contents $n_{d}$ and $n_{p}$ as determined with NMR (r.h.s.) equals the inherent $\mathrm{Cu} 3 d^{9}$ hole content plus the hole content added by doping $x$ (l.h.s.). This agreement was shown to apply for electron, as well as hole doping, and different parent materials differ only in terms of the charge transfer between $\mathrm{Cu}$ and $\mathrm{O}^{19}$. One can therefore also infer from Fig. 1d that compounds with the highest maximum $T_{c}$ favour a smaller $\mathrm{Cu}$ hole content, and we conclude that it is the transfer of hole density to the $\mathrm{O}$ sites that is important for the highest $T_{c}$. With this result, one may ask if other properties of the cuprates should be discussed in terms of $n_{p}$ and $n_{d}$, as well? This leads us to propose a cuprate phase diagram based on NMR.

Phase diagram of the cuprates based on NMR. In Fig. 2 we plot $T_{c}$ as a function of $n_{d}$ and $2 n_{p}$ for all cuprates for which we could find both, $\mathrm{Cu}$ and $\mathrm{O}$ quadrupole splittings in the literature (see Supplementary Tables 1 and 2) with $n_{d}$ and $n_{p}$ calculated from equations (1) and (2). All materials appear in four separate groups, marked by colour: (1) $\mathrm{La}_{2}-{ }_{x} \mathrm{Sr}_{x} \mathrm{CuO}_{4}$; (2) $\mathrm{YBa}_{2} \mathrm{Cu}_{3} \mathrm{O}_{6+y}$, and other cuprates of that structure, for example, $\left(\mathrm{Ca}_{x} \mathrm{La}_{1-x}\right)\left(\mathrm{Ba}_{1.75-x} \mathrm{La}_{0.25+x}\right) \mathrm{Cu}_{3} \mathrm{O}_{6+y}$ as well as $\mathrm{YBa}_{2} \mathrm{Cu}_{4} \mathrm{O}_{8}$; (3) $\mathrm{Bi}$, Tl and $\mathrm{Hg}$ based families; and finally, (4) the two electron-doped systems $\mathrm{Pr}_{2-x} \mathrm{Ce}_{x} \mathrm{CuO}_{4}$ and $\mathrm{Nd}_{2-x} \mathrm{Ce}_{x} \mathrm{CuO}_{4}$. The parent line, that is, the line that is given by $n_{d}+2 n_{p}=1$ (bold dashed line) separates hole doped and electron-doped systems. Note that the lines parallel to the parent line are given by $n_{d}+2 n_{p}=1+x$, and represent constant hole $(x=+0.1$, $+0.2)$ or electron $(x=-0.1,-0.2)$ doping. While there may be material-specific uncertainties, for example, $\mathrm{La}_{2} \mathrm{CuO}_{4}$ is not located exactly on the parent line, our straightforward analysis uncovers simple systematic trends concerning all cuprates, and we discuss some salient features now.

While $n_{d}$ and $n_{p}$ change significantly between different parent compounds along the line $n_{d}+2 n_{p}=1$, antiferromagnetism persists as long as there is one hole per $\mathrm{CuO}_{2}$. Such a large range of variation in the charge transfer in different parent

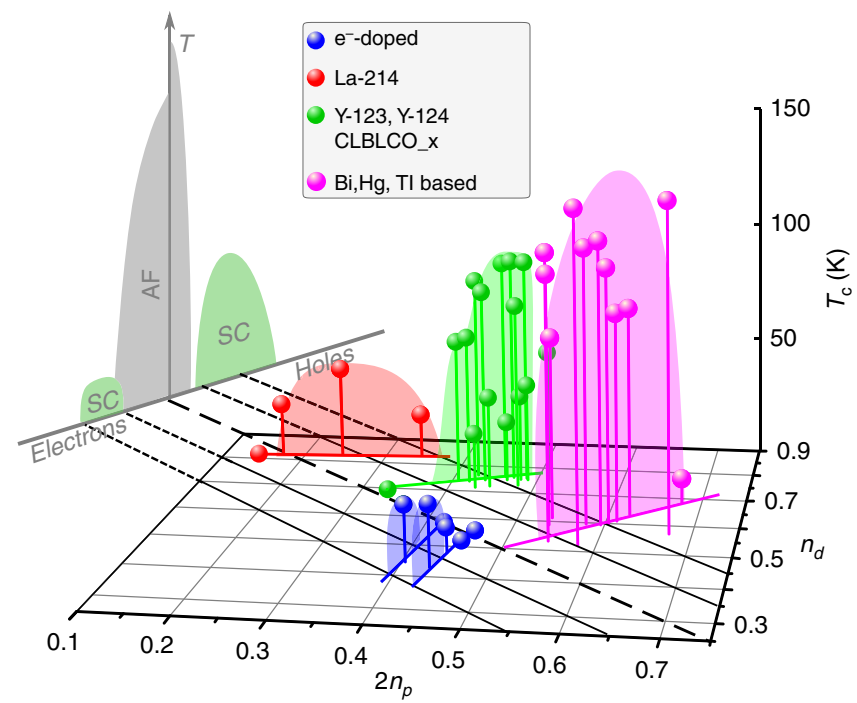

Figure 2 | Cuprate phase diagram from NMR. $T_{\mathrm{c}}$ as a function of oxygen $\left(2 n_{p}\right)$ and copper $\left(n_{d}\right)$ hole content for hole doped La-214, Y-123, Y-124, CLBLCO_x and Bi-, Hg-, Tl-based compounds, as well as electron ( $\mathrm{e}^{-}$) doped $\operatorname{Pr}-214$ and Nd-214. For list of abbreviations see Table 1. The parent line (dashed bold black) indicates expectation for the undoped case $\left(n_{d}+2 n_{p}=1\right.$ from $x=0$ ), parallel lines (thin black) correspond to expectation for doping $x=n_{d}+2 n_{p}-1$ changing with a step of 0.1 . The commonly used phase diagram ( $T$ versus $x$ ) appears as a projection (upper left). compounds, with $2 n_{p}$ ranging from 0.15 to 0.45 , is perhaps quite surprising, and its further increase, if possible, could raise $T_{\mathrm{c}}$ substantially.

Doping holes means entering the right upper half of the $\left(2 n_{p}, n_{d}\right)$-plane. While $n_{d}$ and $n_{p}$ increase with doping, the ratio of the respective changes $\left(\Delta n_{d} / 2 \Delta n_{p}\right)$ appears to be a family property, that is, parent materials with low $n_{p}$ (for example, $\mathrm{La}_{2} \mathrm{CuO}_{4}$ ) add more holes to $\mathrm{O}$ than those with high $n_{p}$. With electron doping we enter the lower left half of the $\left(2 n_{p}, n_{d}\right)$-plane. Here, predominantly $\mathrm{Cu}$ holes disappear while the (large) O hole content changes only slightly. It is not apparent from the phase diagram why most parent materials can only be doped with one type of carrier. As a function of doping, $T_{c}$ increases with a slope that depends on the position on the parent line, as well, and hole doping seems to be more effective in raising $T_{\mathrm{c}}$. Another important observation concerns optimal doping, that is, the doping level for which one finds the highest $T_{\mathrm{c}}$ for a given family. According to our analysis it is related to $x=n_{d}+2 n_{p}-1$ and not particular values of $n_{d}$ and $n_{p}$. However, we do observe a slight increase of the optimal $x$ with increasing $n_{p}$ (decreasing $n_{d}$ ). Note that the doping level $x$ follows from our analysis in terms of $n_{d}$ and $n_{p}$ inserted into (3) and is not deduced from material chemistry. Our analysis agrees with expectations also for materials doped by interstitial $\mathrm{O}_{\delta}$ where doping level $x$ is often derived from the $T_{c}$ dome ${ }^{19}$. Interestingly, the latter materials we find located in the same group, despite significant structural differences between $\mathrm{Hg}$-, $\mathrm{Tl}-$ and Bi-based cuprates. Also the number of close $\mathrm{CuO}_{2}$ layers in multi-layer systems does not result in significant differences in the charge distribution.

\section{Discussion}

In Fig. 2 we plotted only $T_{c}$ in the $\left(2 n_{p}, n_{d}\right)$-phase diagram, but it might be of great interest to investigate whether other cuprate properties are better presented as a function of the local charge distribution, instead of the average doping level. While further analysis is beyond the scope of our paper, we shortly discuss some other cuprate properties with regard to our phase diagram.

The Néel temperature depends on the interlayer coupling and therefore is not expected to be dominated by the charge distribution in the $\mathrm{CuO}_{2}$ plane. For example, $\mathrm{YBa}_{2} \mathrm{Cu}_{3} \mathrm{O}_{6}$ has a higher $T_{N}$ than $\mathrm{Pr}_{2} \mathrm{CuO}_{4}$ and $\mathrm{La}_{2} \mathrm{CuO}_{4}$. It would be interesting, however, to find out how the exchange coupling $(J)$ changes along the parent line. Recently, there have been contradicting reports regarding $J$ in the cuprates ${ }^{57-59}$. Mallet et al. ${ }^{58}$ found no correlation between $J$ and $T_{c \text {, max }}$ in $\mathrm{R}(\mathrm{Ba}, \mathrm{Sr})_{2} \mathrm{Cu}_{3} \mathrm{O}_{y}$, while Wulferding et al. ${ }^{57}$ claimed that $J$ is correlated with $T_{c, \max }$ in $\left(\mathrm{Ca}_{x} \mathrm{La}_{1-x}\right)\left(\mathrm{Ba}_{1.75-x} \mathrm{La}_{0.25+x}\right) \mathrm{Cu}_{3} \mathrm{O}_{6+y}$, which was later questioned by Tallon ${ }^{59}$.

Structural parameters of the $\mathrm{CuO}_{2}$ plane such as distances, buckling, or disorder appear to show no clear trend with respect to $n_{d}$ and $n_{p}$. However, the apical oxygen distance from the $\mathrm{CuO}_{2}$ plane increases as one follows the parent line beginning from low $n_{p}$, similar to the maximum possible $T_{\mathrm{c}}$. This behaviour and the concomitant change in density of states of $\mathrm{Cu} 4 s$ was noted before ${ }^{60}$.

Pressure applied to underdoped cuprates usually increases $T_{c}$, while the structural changes to even hydrostatic pressure can be complicated ${ }^{61}$. For example, specifically strained $\mathrm{HgBa}_{2} \mathrm{CuO}_{4+\delta}$ can have almost identical $\mathrm{CuO}_{6}$ octahedra as $\mathrm{La}_{2}{ }_{x} \mathrm{Sr}_{x} \mathrm{CuO}_{4}$, however, the large difference in their $T_{c}$ values remains ${ }^{62}$. This might be related to the different values of $n_{d}$ and $n_{p}$ for these families. As a result of recent progress in anvil cell NMR ${ }^{63,64}$, it is now possible to study cuprates at high pressures also with $\mathrm{NMR}^{65}$, and it was found that the $\mathrm{Cu}$ splitting increases with pressure indicating changes in the planar hole contents ${ }^{66}$. 
However, single-crystal studies are necessary and ongoing efforts by our group aim at providing a quantitative measure of the local charge distribution as a function of pressure.

Another important issue concerns the heterogeneity of the cuprates. We know from NMR that the static charge and spin density can vary drastically within the $\mathrm{CuO}_{2}$ plane, in particular between different cuprate families ${ }^{67}$. For example, the charge density in terms of the total doping $x$ may easily vary by $\Delta x \approx 0.05$ (refs $32,68,69)$. Since $T_{\mathrm{c}}$ is not in a simple relation to this static inhomogeneity, only the average $n_{d}$ and $n_{p}$ appear to matter. From this, one would conclude that inhomogeneity is either not important for the maximum $T_{\mathcal{C}}$, or it is ubiquitous and dynamically averaged for NMR, depending on the chemical environment.

To conclude, NMR measures the charge distribution in the bonding orbitals in the $\mathrm{CuO}_{2}$ plane quantitatively, and since it reproduces the Uemura plot, that is, it finds the same ordering of families with respect to their maximum $T_{c}$, we now have material chemistry parameters that are responsible for setting the highest $T_{\mathrm{c}}$ and superfluid density. These findings inspired a different perspective on the cuprate phase diagram and it is likely that the complex cuprate properties might be better understood when discussed in the context of the charge distribution in the $\mathrm{CuO}_{2}$ plane.

\section{References}

1. Uemura, Y. J. et al. Universal correlations between $T_{c}$ and $n_{s} / m^{*}$ (carrier density over effective mass) in high- $T_{c}$ cuprate superconductors. Phys. Rev. Lett. 62, 2317-2320 (1989).

2. Savici, A. T. et al. Muon spin relaxation studies of incommensurate magnetism and superconductivity in stage- $4 \mathrm{La}_{2} \mathrm{CuO}_{4.11}$ and $\mathrm{La}_{1.88} \mathrm{Sr}_{0.12} \mathrm{CuO}_{4}$. Phys. Rev. $B$ 66, 014524 (2002)

3. Homes, C. C. et al. A universal scaling relation in high-temperature superconductors. Nature 430, 539-541 (2004).

4. Tallon, J. L., Cooper, J. R., Naqib, S. H. \& Loram, J. W. Scaling relation for the superfluid density of cuprate superconductors: origins and limits. Phys. Rev. B 73, 180504 (2006)

5. Homes, C. C. Scaling of the superfluid density in strongly underdoped $\mathrm{YBa}_{2} \mathrm{Cu}_{3} \mathrm{O}_{6+y}$ : evidence for a Josephson phase. Phys. Rev. B 80, 180509 (2009).

6. Dordevic, S. V., Basov, D. N. \& Homes, C. C. Do organic and other exotic superconductors fail universal scaling relations? Sci. Rep. 3, 1713 (2013).

7. Wu, D. et al. Superfluid density of $\mathrm{Ba}\left(\mathrm{Fe}_{1-x} \mathrm{M}_{x}\right)_{2} \mathrm{As}_{2}$ from optical experiments. Phys. C Supercond. 470, S399-S400 (2010).

8. Homes, C. C., Xu, Z. J., Wen, J. S. \& Gu, G. D. Effective medium approximation and the complex optical properties of the inhomogeneous superconductor $\mathrm{K}_{0.8} \mathrm{Fe}_{2-y} \mathrm{Se}_{2}$. Phys. Rev. B 86, 144530 (2012).

9. Shengelaya, A. \& Müller, K. A. The intrinsic heterogeneity of superconductivity in the cuprates. EPL 109, 27001 (2015).

10. Imry, Y., Strongin, M. \& Homes, C. C. $n_{s}-T_{c}$ correlations in granular superconductors. Phys. Rev. Lett. 109, 067003 (2012).

11. Kogan, V. G. Homes scaling and BCS. Phys. Rev. B 87, 220507 (R) (2013).

12. Lindner, M. H. \& Auerbach, A. Conductivity of hard core bosons: a paradigm of a bad metal. Phys. Rev. B 81, 054512 (2010).

13. Haase, J., Slichter, C. \& Milling, C. Static charge and spin inhomogeneity in $\mathrm{La}_{2}{ }_{x} \mathrm{Sr}_{x} \mathrm{CuO}_{4}$ by NMR. J. Supercond. 15, 339-343 (2002).

14. Ofer, R., Levy, S., Kanigel, A. \& Keren, A. Charge-inhomogeneity doping relations in $\mathrm{YBa}_{2} \mathrm{Cu}_{3} \mathrm{O}_{y}$ detected by angle-dependent nuclear quadrupole resonance. Phys. Rev. B 73, 012503 (2006).

15. Schwarz, K., Ambrosch-Draxl, C. \& Blaha, P. Charge distribution and electric-field gradients in $\mathrm{YBa}_{2} \mathrm{Cu}_{3} \mathrm{O}_{7-x}$. Phys. Rev. B 42, 2051-2061 (1990).

16. Zheng, G., Kitaoka, Y., Ishida, K. \& Asayama, K. Local hole distribution in the $\mathrm{CuO}_{2}$ plane of high- $T_{\mathrm{c}} \mathrm{Cu}$-oxides studied by $\mathrm{Cu}$ and oxygen NQR/NMR. J. Phys. Soc. Jpn 64, 2524-2532 (1995)

17. Stoll, E. P., Meier, P. F. \& Claxton, T. A. Electric field gradients from first-principles and point-ion calculations. Phys. Rev. B 65, 064532 (2002).

18. Haase, J., Sushkov, O. P., Horsch, P. \& Williams, G. Planar $\mathrm{Cu}$ and O hole densities in high- $T_{c}$ cuprates determined with NMR. Phys. Rev. B 69, 0945041 (2004).

19. Jurkutat, M. et al. Distribution of electrons and holes in cuprate superconductors as determined from ${ }^{17} \mathrm{O}$ and ${ }^{63} \mathrm{Cu}$ nuclear magnetic resonance. Phys. Rev. B 90, 140504 (2014).
20. Ishida, K., Kitaoka, Y., Asayama, K., Kadowaki, K. \& Mochiku, T. Cu NMR study in single crystal $\mathrm{Bi}_{2} \mathrm{Sr}_{2} \mathrm{CaCu}_{2} \mathrm{O}_{8}$ observation of gapless superconductivity. J. Phys. Soc. Jpn 63, 1104-1113 (1994).

21. Fujiwara, K. et al. NMR and NQR studies of superconductivity in heavily doped $\mathrm{Tl}_{2} \mathrm{Ba}_{2} \mathrm{CuO}_{6+y}$ with a single $\mathrm{CuO}_{2}$ plane. Phys. C Supercond. 184, 207-219 (1991).

22. Magishi, K. et al. Magnetic excitation and superconductivity in overdoped $\mathrm{TlSr}_{2} \mathrm{CaCu}_{2} \mathrm{O}_{7-\delta}$ : a ${ }^{63} \mathrm{Cu}$ NMR study. Phys. Rev. B 54, 10131-10142 (1996).

23. Goto, T., Nakajima, S., Kikuchi, M., Syono, Y. \& Fukase, T. ${ }^{63 / 65} \mathrm{Cu}$ and 203/205 Tl NMR study on the antiferromagnetic phase of the Tl-based high- $T_{c}$ oxide $\mathrm{TlBa}_{2} \mathrm{YCu}_{2} \mathrm{O}_{7}$. Phys. Rev. B 54, 3562-3570 (1996).

24. Gerashenko, A. et al. The ${ }^{63} \mathrm{Cu}$ and ${ }^{17} \mathrm{O}$ NMR studies of spin susceptibility in differently doped $\mathrm{Tl}_{2} \mathrm{Ba}_{2} \mathrm{CaCu}_{2} \mathrm{O}_{8-\delta}$ compounds. Phys. C. Supercond. 328, 163-176 (1999).

25. Han, Z. P., Dupree, R., Liu, R. \& Edwards, P. ${ }^{63} \mathrm{Cu}$ NMR shift and relaxation behavior in $\mathrm{Tl}_{2} \mathrm{Ba}_{2} \mathrm{Ca}_{2} \mathrm{Cu}_{3} \mathrm{O}_{10-\delta}\left(T_{c}=125 \mathrm{~K}\right)$. Phys. C. Supercond. 226, 106-112 (1994).

26. Williams, G. V. M., Krämer, S. \& Mehring, M. Nuclear-quadrupole-resonance study of overdoped $\mathrm{Y}_{1-x} \mathrm{Ca}_{x} \mathrm{Ba}_{2} \mathrm{Cu}_{3} \mathrm{O}_{7}$. Phys. Rev. B 63, 104514 (2001).

27. Zheng, G. et al. NMR study of local hole distribution, spin fluctuation and superconductivity in $\mathrm{Tl}_{2} \mathrm{Ba}_{2} \mathrm{Ca}_{2} \mathrm{Cu}_{3} \mathrm{O}_{10}$. Phys. C. Supercond. 260, 197-210 (1996).

28. Gippius, A. A., Antipov, E. V., Hoffmann, W. \& Luders, K. Nuclear quadrupole interactions and charge localization in $\mathrm{HgBa}_{2} \mathrm{CuO}_{4}+\delta$ with different oxygen content. Phys. C. Supercond. 276, 57-64 (1997).

29. Shimizu, S. et al. Planar $\mathrm{CuO}_{2}$ hole density in high- $\mathrm{T}_{c}$ cuprates determined by NMR Knight shift: ${ }^{63} \mathrm{Cu}$ NMR on bilayered $\mathrm{Ba}_{2} \mathrm{CaCu}_{2} \mathrm{O}_{4}(\mathrm{~F}, \mathrm{O})_{2}$ and threelayered $\mathrm{Ba}_{2} \mathrm{Ca}_{2} \mathrm{Cu}_{3} \mathrm{O}_{6}(\mathrm{~F}, \mathrm{O})_{2}$. Phys. Rev. B 83, 144523 (2011).

30. Rybicki, D. et al. ${ }^{63} \mathrm{Cu}$ and ${ }^{199} \mathrm{Hg} \mathrm{NMR}$ study of $\mathrm{HgBa}_{2} \mathrm{CuO}_{4+\delta}$ single crystals, Preprint at: http://arxiv.org/abs/1208.4690 (2012).

31. Keren, A., Kanigel, A. \& Bazalitsky, G. Evidence for two fluids in cuprate superconductors from a nuclear resonance study of $\left(\mathrm{Ca}_{x} \mathrm{La}_{1-x}\right)\left(\mathrm{Ba}_{1.75-x} \mathrm{La}_{0.25+x}\right) \mathrm{Cu}_{3} \mathrm{O}_{y}$. Phys. Rev. B 74, 172506 (2006).

32. Rybicki, D. et al. Spatial inhomogeneities in single-crystal $\mathrm{HgBa}_{2} \mathrm{CuO}_{4+\delta}$ from ${ }^{63} \mathrm{Cu}$ NMR spin and quadrupole shifts. J. Supercond. Nov. Magn. 22, 179-183 (2009).

33. Ohsugi, S., Tsuchiya, T., Koyama, T. \& Fueki, K. Gapless superconductivity in overdoped Hg system; Cu-NQR study. J. Low Temp. Phys. 105, 419-423 (1996).

34. Horvatić, M. et al. NMR investigation of $\mathrm{HgBa}_{2} \mathrm{CaCu}_{2} \mathrm{O}_{6+\delta}$. Phys. C. Supercond. 235, 1669-1670 (1994).

35. Julien, M.-H. et al. ${ }^{63} \mathrm{Cu}$ and ${ }^{199} \mathrm{Hg} \mathrm{NMR}$ in overdoped $\mathrm{HgBa}_{2} \mathrm{CaCu}_{2} \mathrm{O}_{6+\delta}$. Phys. C. Supercond. 268, 197-204 (1996).

36. Julien, M.-H., Horvatić, M., Berthier, C. \& Segransan, P. ${ }^{63} \mathrm{Cu}$ NMR in the normal state of $\mathrm{HgBa}_{2} \mathrm{Ca}_{2} \mathrm{Cu}_{3} \mathrm{O}_{8+\delta}$. J. Low Temp. Phys. 105, 371-376 (1996).

37. Magishi, K. et al. ${ }^{63} \mathrm{Cu}$ NMR probe of superconducting properties in $\mathrm{HgBa}_{2} \mathrm{Ca}_{2} \mathrm{Cu}_{3} \mathrm{O}_{8+\delta}$ : a possible reason for $T_{c}=133 \mathrm{~K}$. Phys. Rev. B 53, R8906-R8909 (1996).

38. Breitzke, H., Eremin, I., Manske, D., Antipov, E. \& Luders, K. Formation of magnetic moments in the cuprate superconductor $\mathrm{Hg}_{0.8} \mathrm{Cu}_{0.2} \mathrm{Ba}_{2} \mathrm{Ca}_{2} \mathrm{Cu}_{3} \mathrm{O}_{8+\delta}$ below $\mathrm{T}_{c}$ seen by NQR. Phys. C. Supercond. 406, 27-36 (2004).

39. Mikhalev, K. et al. ${ }^{63} \mathrm{Cu}$ NMR study of infinite-layer compound $\mathrm{Sr}_{1-x} \mathrm{La}_{x} \mathrm{CuO}_{2}$. Phys. C. Supercond. 304, 165-171 (1998).

40. Itohara, $\mathrm{K}$. et al. Number of $\mathrm{CuO}_{2}$ layers dependence of magnetic quantum criticality in homogeneously doped high- $T_{c}$ copper oxides: a ${ }^{63} \mathrm{Cu}-\mathrm{NMR}$ study on four-layered high- compounds $\mathrm{HgBa}_{2} \mathrm{Ca}_{3} \mathrm{Cu}_{4} \mathrm{O}_{8+y}$. Phys. C. Supercond. 470, S140-S141 (2010).

41. Imai, T., Slichter, C., Cobb, J. \& Markert, J. Superconductivity and spin fluctuations in the electron-doped infinitely-layered high $T_{c}$ superconductor $\mathrm{Sr}_{0.9} \mathrm{La}_{0.1} \mathrm{CuO}_{2}\left(T_{c}=42 \mathrm{~K}\right)$. J. Phys. Chem. Solids 56, 1921-1925 (1995).

42. Kotegawa, $\mathrm{H}$. et al. Coexistence of superconductivity and antiferromagnetism in multilayered high- $T_{c}$ superconductor $\mathrm{HgBa}_{2} \mathrm{Ca}_{4} \mathrm{Cu}_{5} \mathrm{O}_{y}$ : $\mathrm{Cu}-\mathrm{NMR}$ study. Phys. Rev. B 69, 014501 (2004).

43. Mounce, A. M. et al. Absence of static loop-current magnetism at the apical oxygen site in $\mathrm{HgBa}_{2} \mathrm{CuO}_{4+\delta}$ from NMR. Phys. Rev. Lett. 111, 187003 (2013).

44. Kambe, S., Yasuoka, H., Hayashi, A. \& Ueda, Y. NMR study of the spin dynamics in $\mathrm{Tl}_{2} \mathrm{Ba}_{2} \mathrm{CuO}_{y}\left(T_{c}=85 \mathrm{~K}\right)$. Phys. Rev. B 47, 2825-2834 (1993).

45. Takigawa, M. \& Mitzi, D. B. NMR studies of spin excitations in superconducting $\mathrm{Bi}_{2} \mathrm{Sr}_{2} \mathrm{CaCu}_{2} \mathrm{O}_{8+\delta}$ single crystals. Phys. Rev. Lett. 73, 1287-1290 (1994).

46. Crocker, J. et al. NMR studies of pseudogap and electronic inhomogeneity in $\mathrm{Bi}_{2} \mathrm{Sr}_{2} \mathrm{CaCu}_{2} \mathrm{O}_{8+\delta}$. Phys. Rev. B 84, 224502 (2011).

47. Trokiner, A. et al. ${ }^{17} \mathrm{O}$ NMR in high- $T_{c}$ superconductor $\mathrm{Tl}_{2} \mathrm{Ba}_{2} \mathrm{CaCu}_{2} \mathrm{O}_{y}$. Phys. C. Supercond. 255, 204-210 (1995).

48. Lim, K., Lee, H. \& Hur, N. An ${ }^{17} \mathrm{O}$ NMR study of $\mathrm{Hg}_{0.5} \mathrm{Tl}_{0.5} \mathrm{Ba}_{2}\left(\mathrm{Ca}_{1-x} \mathrm{Sr}_{x}\right)_{2}$ $\mathrm{Cu}_{3} \mathrm{O}_{8+\delta}$. Phys. C. Supercond. 232, 215-221 (1994).

49. Amit, E. \& Keren, A. Critical-doping universality for cuprate superconductors: Oxygen nuclear-magnetic-resonance investigation of $\left(\mathrm{Ca}_{x} \mathrm{La}_{1-x}\right)\left(\mathrm{Ba}_{1.75-x} \mathrm{La}_{0.25+x}\right) \mathrm{Cu}_{3} \mathrm{O}_{y}$. Phys. Rev. B 82, 172509 (2010). 
50. Uemura, Y. J. et al. Magnetic-field penetration depth in $\mathrm{Tl}_{2} \mathrm{Ba}_{2} \mathrm{CuO}_{6+\delta}$ in the overdoped regime. Nature 364, 605-607 (1993).

51. Niedermayer, C. et al. Muon spin rotation study of the correlation between $T_{c}$ and $n_{s} / m^{*}$ in overdoped $\mathrm{Tl}_{2} \mathrm{Ba}_{2} \mathrm{CuO}_{6+\delta}$. Phys. Rev. Lett. 71, 1764-1767 (1993).

52. Tallon, J. L., Bernhard, C. \& Niedermayer, C. Muon spin relaxation studies of superconducting cuprates. Supercond. Sci. Technol. 10, A38-A51 (1997).

53. Shengelaya, A. et al. Muon-spin-rotation measurements of the penetration depth of the infinite-layer electron-doped $\mathrm{Sr}_{0.9} \mathrm{La}_{0.1} \mathrm{CuO}_{2}$ cuprate superconductor. Phys. Rev. Lett. 94, 127001 (2005).

54. Homes, C. C., Clayman, B. P., Peng, J. L. \& Greene, R. L. Optical properties of $\mathrm{Nd}_{1.85} \mathrm{Ce}_{0.15} \mathrm{CuO}_{4}$. Phys. Rev. B 56, 5525-5534 (1997).

55. Azuma, M., Hiroi, Z., Takano, M., Bando, Y. \& Takeda, Y. Superconductivity at $110 \mathrm{~K}$ in the infinite-layer compound $\left(\mathrm{Sr}_{1-x} \mathrm{Ca}_{x}\right)_{1-y} \mathrm{CuO}_{2}$. Nature 356, 775-776 (1992).

56. Chu, C., Deng, L. \& Lv, B. Hole-doped cuprate high temperature superconductors. Phys. C. Supercond. 514, 290-313 (2015).

57. Wulferding, D. et al. Relation between cuprate superconductivity and magnetism: a Raman study of $(\mathrm{CaLa})_{1}(\mathrm{BaLa})_{2} \mathrm{Cu}_{3} \mathrm{O}_{y}$. Phys. Rev. B 90, 104511 (2014).

58. Mallett, B. P. P. et al. Dielectric versus magnetic pairing mechanisms in hightemperature cuprate superconductors investigated using Raman scattering. Phys. Rev. Lett. 111, 237001 (2013).

59. Tallon, J. L. Anomalous behavior of $T_{c}$ and pseudogap in the superconductor $\mathrm{Ca}_{x} \mathrm{La}_{1-x} \mathrm{Ba}_{1.75-x} \mathrm{La}_{0.25+x} \mathrm{Cu}_{3} \mathrm{O}_{y}$ with respect to doping and ion size. Phys. Rev. B 90, 214523 (2014).

60. Pavarini, E., Dasgupta, I., Saha-Dasgupta, T., Jepsen, O. \& Andersen, O. K. Band-structure trend in hole-doped cuprates and correlation with $T_{c, \text { max }}$. Phys. Rev. Lett. 87, 047003 (2001).

61. Schilling, J. S. in High pressure effects in Handbook of High Temperature Superconductivity: Theory and Experiment (eds Schrieffer, J. \& Brooks, J.) (Springer Verlag, 2007).

62. Wang, S. et al. Strain derivatives of $T_{c}$ in $\mathrm{HgBa}_{2} \mathrm{CuO}_{4+\delta}$ : the $\mathrm{CuO}_{2}$ plane alone is not enough. Phys. Rev. B 89, 024515 (2014).

63. Haase, J., Goh, S. K., Meissner, T., Alireza, P. \& Rybicki, D. High sensitivity nuclear magnetic resonance probe for anvil cell pressure experiments. Rev. Sci. Intrum. 80, 073905 (2009).

64. Meissner, T. et al. New approach to high-pressure nuclear magnetic resonance with anvil cells. J. Low Temp. Phys. 159, 284-287 (2010).

65. Meissner, T., Goh, S., Haase, J., Williams, G. V. M. \& Littlewood, P. B. Highpressure spin shifts in the pseudogap regime of superconducting $\mathrm{YBa}_{2} \mathrm{Cu}_{4} \mathrm{O}_{8}$ as revealed by ${ }^{17} \mathrm{O}$ NMR. Phys. Rev. B 83, 220517 (2011).

66. Meissner, T. Exploring nuclear magnetic resonance at the highest pressures closing the pseudogap under pressure in a high temperature superconductor. $\mathrm{PhD}$ thesis Univ. Leipzig (2012).
67. Haase, J. Charge density variation in $\mathrm{YBa}_{2} \mathrm{Cu}_{3} \mathrm{O}_{6+y}$. Phys. Rev. Lett. 91, 189701 (2003).

68. Jurkutat, M., Haase, J. \& Erb, A. Charge inhomogeneity in electron-doped $\operatorname{Pr}_{1.85} \mathrm{Ce}_{0.15} \mathrm{CuO}_{4}$ determined with ${ }^{63} \mathrm{Cu}$ NMR. J. Supercond. Nov. Magn. 26, 2685-2688 (2013).

69. Singer, P. M., Hunt, A. W. \& Imai, T. ${ }^{63} \mathrm{Cu}$ NQR evidence for spatial variation of hole concentration in $\mathrm{La}_{2}{ }_{x} \mathrm{Sr}_{x} \mathrm{CuO}_{4}$. Phys. Rev. Lett. 88, 047602 (2002).

\section{Acknowledgements}

We are thankful to O.P. Sushkov, D. K. Morr, C.P. Slichter, G.V.M. Williams for helpful discussions, and acknowledge the financial support by the University of Leipzig, the DFG within the Graduate School Build-MoNa, the European Social Fund (ESF), the Free State of Saxony, and the Ministry of Science and Higher Education of Poland.

\section{Author contributions}

D.R., M.J. and S.R. contributed to data gathering, analysis and writing the manuscript. All the authors discussed the results and worked on the manuscript. C.K. helped with discussion of the $\mu \mathrm{SR}$ technique and its relation to NMR. J.H. supervised and contributed equally to the data analysis and manuscript editing, as well as providing project leadership.

\section{Additional information}

Supplementary Information accompanies this paper at http://www.nature.com/ naturecommunications

Competing financial interests: The authors declare no competing financial interests.

Reprints and permission information is available online at http://npg.nature.com/ reprintsandpermissions/

How to cite this article: Rybicki, D. et al. Perspective on the phase diagram of cuprate high-temperature superconductors. Nat. Commun. 7:11413 doi: 10.1038/ncomms11413 (2016).

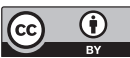

This work is licensed under a Creative Commons Attribution 4.0 International License. The images or other third party material in this article are included in the article's Creative Commons license, unless indicated otherwise in the credit line; if the material is not included under the Creative Commons license, users will need to obtain permission from the license holder to reproduce the material. To view a copy of this license, visit http://creativecommons.org/licenses/by/4.0/ 